Du 15 au 17 mars, MM. Hay et Sommaruga, accompagnés de M. Michel Veuthey, délégué général pour l'Europe et l'Amérique du Nord, se sont rendus à Rome où ils ont été reçus en audience privée par S.S. le Pape Jean-Paul II. Ils ont également rencontré le Cardinal Casaroli, secrétaire d'Etat, Mgr E. Martinez Somalo, substitut de la secrétairerie d'état et Mgr A. Silvestrini, secrétaire d'état pour les Affaires publiques de l'Eglise.

$\mathrm{Au}$ Ministère italien des Affaires étrangères, les représentants du CICR ont eu des entretiens avec M. Giulio Andreotti, ministre des Affaires étrangères, l'ambassadeur Renato Ruggiero, secrétaire général, l'ambassadeur Patrizio Schmidlin, directeur général du département pour la Coopération au Développement, l'ambassadeur Boris Biancheri, directeur général du département des Affaires politiques, en présence de l'ambassadeur Franceschi, représentant permanent de l'Italie à Genève.

Cette double visite présidentielle à Rome s'inscrivait dans le cadre des efforts déployés par le CICR pour mieux faire connaître aux Etats parties aux Conventions de Genève ses activités dans le monde et leur faire partager ses préoccupations face aux violations graves des normes humanitaires dans plusieurs conflits armés. M. Hay eut également l'occasion d'exprimer aux Autorités italiennes la gratitude du CICR pour le soutien financier que celles-ci lui apportent depuis plusieurs années, confiant que ce soutien sera confirmé, et si possible renforcé à l'avenir.

Lors de leur séjour à Rome, MM. Hay et Sommaruga eurent aussi des entretiens avec des représentants de la Croix-Rouge italienne, le Dr. Ugo Ciantelli, vice-commissaire, le professeur Renato Pons, directeur général, Mme Manuela Lavagnino, chef du service des Affaires internationales, Mme Mariapia Fanfani, vice-présidente de la Ligue et présidente du comité national féminin de la Croix-Rouge italienne.

\title{
Décès de M. Michel Testuz
}

C'est avec une profonde tristesse que le CICR a appris, le 2 avril écoulé, le décès de M. Michel Testuz, ancien rédacteur de la Revue, des suites de l'infarctus qui l'avait tenu éloigné du CICR depuis l'automne 1985. 
M. Testuz a commencé sa carrière en 1948 déjà, alors qu'il se spécialisait à l'Ecole archéologique de Jérusalem, ville où il fut contacté pour collaborer avec la mission du CICR.

De 1950 à 1960, il devait d'abord poursuivre ses études, puis enseigner la langue et la littérature hébraïques aux universités de Rome, Lausanne et Genève. Dans cette dernière ville, tout en étant chargé de cours, il assuma également, durant l'année 1960, la direction de la Bibliothèque Bodmer. Durant cette même période, il publia une dizaine de volumes scientifiques, des articles de revues, etc.

En 1960, il rejoignit le CICR pour être envoyé au Japon avec le titre de chef de délégation, poste qu'il occupa jusqu'en 1968. De 1968 à 1970, il fut chef de la délégation au Cambodge, période durant laquelle il effectua également de nombreuses missions dans diflerents pays voisins.

A partir de 1970, il devait rejoindre Genève pour occuper le poste d'adjoint du délégué général pour l'Asie. Les années 1971 à 1973 le virent à nouveau sur le terrain comme chef de la délégation au Pakistan. En 1976 enfin, il assuma la responsabilité de la délégation du Caire.

A la suite du départ à la retraite de M. Jean-Georges Lossier, M. Testuz assuma, dès 1977, avec compétence, le poste de rédacteur de la Revue, fonction qu'il devait quitter, en automne 1985, pour raison de maladie.

Homme d'une grande culture, véritable humaniste, Michel Testuz restera aussi pour ceux qui l'ont connu, un homme d'une modestie extrême. D'une exquise amabilité avec ceux qu'il côtoyait, il avait su, mieux que personne, durant ses nombreuses missions, deviner chez ses interlocuteurs une sensibilité différente qu'il respectait. Spirituel, drôle avec ses intimes, M. Testuz se gardait bien de ne jamais blesser autrui.

En qualité de rédacteur de la Revue, il maintint à cette dernière une grande rigueur et une qualité que ses lecteurs surent toujours lui reconnaître.

Les collaborateurs du CICR et ses nombreux amis de par le monde garderont de lui le meilleur souvenir. 